\title{
Ultra-low noise microwave signal generation with an optical frequency comb
}

\author{
Xiaopeng Xie, Romain Bouchand, Daniele Nicolodi, \\ Michele Giunta, Wolfgang Hänsel, et al.
}

Xiaopeng Xie, Romain Bouchand, Daniele Nicolodi, Michele Giunta, Wolfgang Hänsel, Matthias Lezius, Abhay Joshi, Shubhashish Datta, Christophe Alexandre, Michel Lours, Pierre-Alain Tremblin, Giorgio Santarelli, Ronald Holzwarth, Yann Le Coq, "Ultra-low noise microwave signal generation with an optical frequency comb," Proc. SPIE 10518, Laser Resonators, Microresonators, and Beam Control XX, 105180N (16 February 2018); doi: $10.1117 / 12.2286316$

SPIE. Event: SPIE LASE, 2018, San Francisco, California, United States 


\title{
Ultra-low noise microwave signal generation with an optical frequency comb
}

Xiaopeng Xie ${ }^{\mathrm{a}}$, Romain Bouchand ${ }^{\mathrm{a}, \dagger}$, Daniele Nicolodi ${ }^{\mathrm{a}, \ddagger}$, Michele Giunta ${ }^{\mathrm{b}, \mathrm{c}}$, Wolfgang Hänsel ${ }^{\mathrm{b}}$, Matthias Lezius $^{\mathrm{b}}$, Abhay Joshid ${ }^{\mathrm{d}}$, Shubhashish Dattad ${ }^{\mathrm{d}}$, Christophe Alexandre ${ }^{\mathrm{e}}$, Michel Lours ${ }^{\mathrm{a}}$, Pierre-Alain Tremblin ${ }^{\mathrm{f}}$, Giorgio Santarelli ${ }^{\mathrm{f}}$, Ronald Holzwarth ${ }^{\mathrm{b}, \mathrm{c}}$, and Yann Le Coq ${ }^{\mathrm{a}}$

${ }^{a}$ LNE-SYRTE, Observatoire de Paris, PSL Research University, CNRS, Sorbonne Universités, UPMC Univ. Paris 06, 61 avenue de I'Observatoire, 75014 Paris,France

bMenlo Systems GmbH, Am Klopferspitz 19a, D-82152 Martinsried, Germany

${ }^{\mathrm{c}}$ Max-Planck-Institut für Quantenoptik, 85748 Garching, Germany

${ }^{d}$ Discovery Semiconductors Inc., 119 Silvia Street, Ewing, New Jersey 08628, USA.

${ }^{\mathrm{e}}$ CNAM, CEDRIC Laboratory, 292 rue Saint Martin, 75003 Paris, France.

${ }^{f}$ LP2N, IOGS CNRS Université de Bordeaux 1, rue F. Mitterand, 33400 Talence, France

${ }^{\dagger}$ Currently with École Polytechnique Fédérale de Lausanne (EPFL), CH-1015 Lausanne, Switzerland

${ }^{\ddagger}$ Currently with National Institute of Standards and Technology, 325 Broadway, Boulder, Colorado 80305, USA

\begin{abstract}
Photonic synthesis of radio frequency waveforms revived the quest for unrivalled microwave purity by its seducing ability to convey the benefits of the optics to the microwave world. In this contribution, we will present a high-fidelity transfer of frequency stability between an optical reference and a microwave signal via a low-noise fiber-based frequency comb and cutting-edge photo-detection techniques. We will show the generation of the purest microwave signal with a fractional frequency stability below $6.5 \times 10^{-16}$ at $1 \mathrm{~s}$ and a timing noise floor below $41 \mathrm{zs} \mathrm{Hz}^{-1 / 2}$ (phase noise below $-173 \mathrm{dBc} \mathrm{Hz}^{-1}$ for a $12 \mathrm{GHz}$ carrier). This outclasses existing sources and promises a new era for state-of-the-art microwave generation. The characterization is achieved through a heterodyne cross-correlation scheme with lowermost detection noise. This unprecedented level of purity can impact domains such as radar systems, telecommunications and time-frequency metrology. The measurements methods developed here can benefit the characterization of a broad range of signals.
\end{abstract}

Keywords: Microwave photonics, phase noise, optical frequency combs, photodiode, precision measurement

\section{INTRODUCTION}

Microwave signals with ultra-low phase noise are used in a great number of fields, such as radar systems, ${ }^{1}$ navigation, long baseline interferometer, telecommunications ${ }^{2}$ and time-frequency metrology. ${ }^{3,4}$ Photonic microwave generation methods, like the optoelectronics oscillator, ${ }^{5}$ Brillouin oscillator, ${ }^{6}$ sideband-injection-locked laser, ${ }^{7}$ electro-optical-frequency division ${ }^{8}$ and Kerr-frequency-comb oscillator, ${ }^{9}$ have attracted a lot of attention in the last two decades as they have very interesting properties, high frequency, large bandwidth, tunability and chip-scale packaging.

In particular, ultra-stable lasers and optical frequency comb based frequency division scheme can produce microwave signals with ultra-low phase noise both in low frequency offset and high frequency offset. ${ }^{3,10-14}$ In this contribution, we will report on photonic microwave generation with unprecedented phase noise result by this scheme and its characterization. ${ }^{14}$ Figure 1 presents the single sideband (SSB) phase noise results comparison of our result with other classic microwave generation methods.

Further author information: (Send correspondence to Yann Le Coq)

Yann Le Coq: E-mail: yann.lecoq@obspm.fr

Laser Resonators, Microresonators, and Beam Control XX, edited by Alexis V. Kudryashov,

Alan H. Paxton, Vladimir S. Ilchenko, Proc. of SPIE Vol. 10518, 105180N · C 2018 SPIE

CCC code: $0277-786 \mathrm{X} / 18 / \$ 18 \cdot$ doi: $10.1117 / 12.2286316$ 


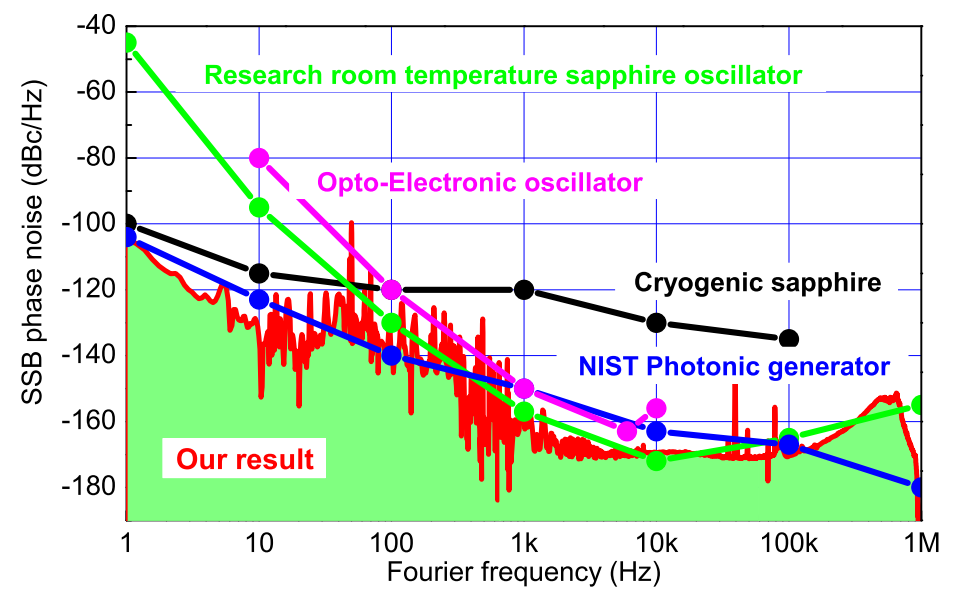

Figure 1. SSB phase noise performance comparison of different microwave generation methods.

\section{MICROWAVE SIGNALS GENERATION WITH AN OPTICAL FREQUENCY COMB}

Optical frequency combs are lasers that emit with a series of evenly spaced ultra-short pulses, corresponding to discrete equidistant lines in the optical spectrum. ${ }^{15,16}$ Phase locking one of these comb lines to an ultra-stable continue-wave $(\mathrm{CW})$ laser reference $\nu_{C W}$ can transfer the spectrum purity of reference to all the other comb lines. The carrier envelop offset (CEO) frequency $f_{0}$ is technically removed during the phase locking process so that the fractional frequency stability of optical reference is transferred to the repetition frequency $f_{r}$ and it harmonics. ${ }^{14}$ Photodetecting these stabilized optical comb lines, harmonics of $f_{r}$ are obtained until the cutoff frequency of photodiode. Their phase noise power spectral density (PSD) is expected to be $\left(\nu_{C W} / f_{\mu}\right)^{2}$ times lower than the phase noise PSD of reference laser, where $f_{\mu}$ is the carrier frequency of harmonics of $f_{r}$.

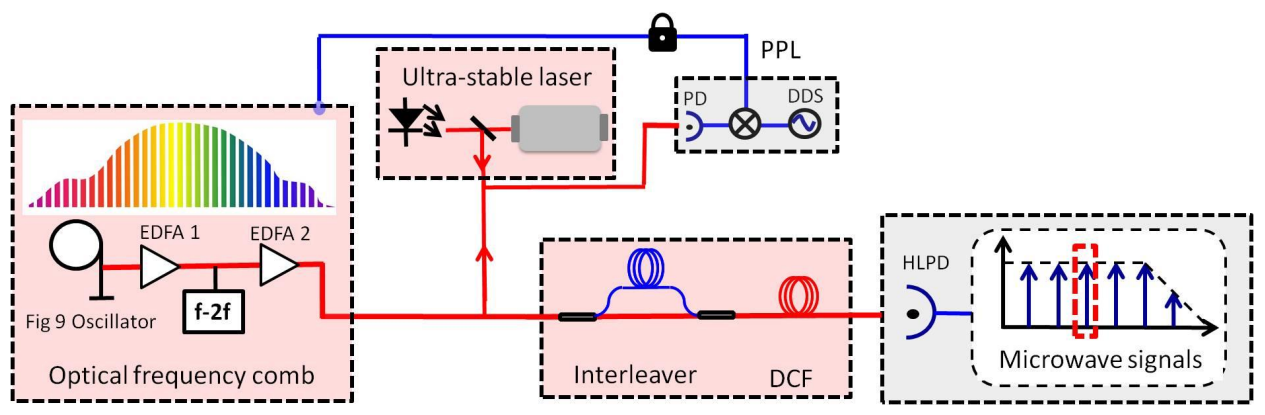

Figure 2. Photonic microwave generation scheme. EDFA, Erbium-doped fiber amplifier; DCF: dispersion compensating fiber; HLPD, High-linear photodiode; PD, Photodiode; DDS, direct digital synthesizer; PPL, Phase lock loop.

Figure 2 shows our photonic microwave generation scheme. An ultra-stable laser with fractional frequency stability as low as $5.5 \times 10^{-16}$ at $1 \mathrm{~s}$ is used as a reference to stabilized the optical frequency comb. The laser phase noise PSD is characterized by a digital cross correlation system as we have reported in Ref. 17.

An ultra-low noise fiber-based optical frequency comb system acts the frequency divider. It consists of three parts: a femtosecond laser, an f-2f interferometer module and erbium-doped fibre amplifiers (EDFA), all made with polarization-maintaining fibre. Based on the nonlinear amplifying loop mirror mode-locking principle, the laser reaches a steady state in a few seconds and can remain in the mode-locked state for several months. The output of femtosecond laser shows a pulse width below $50 \mathrm{fs}$, with an optical spectrum broader than $60 \mathrm{~nm}$. The 
The repetition rate is coarsely tunable over $5 \mathrm{MHz}$ around $250 \mathrm{MHz}$. An intracavity electro-optical modulator (EOM) provides feedback on the comb repetition rate with a bandwidth of roughly $1 \mathrm{MHz} .{ }^{18}$ The laser output is pre-amplified up to $30 \mathrm{~mW}$ by a first EDFA and split into two parts that are used to seed the f-2f interferometer for the CEO-frequency detection and by a second EDFA for microwave generation. The second high-power EDFA boosts the optical power up to $350 \mathrm{~mW}$ and passively reduces the relatively intensity noise (RIN) through seed saturation. It is pumped by three low-noise single-mode high-power laser diodes, all set to specific currents to avoid parasitic mode hopping. For each diode, the lowest RIN is observed when the current set point is at an equal distance from two successive mode hops.

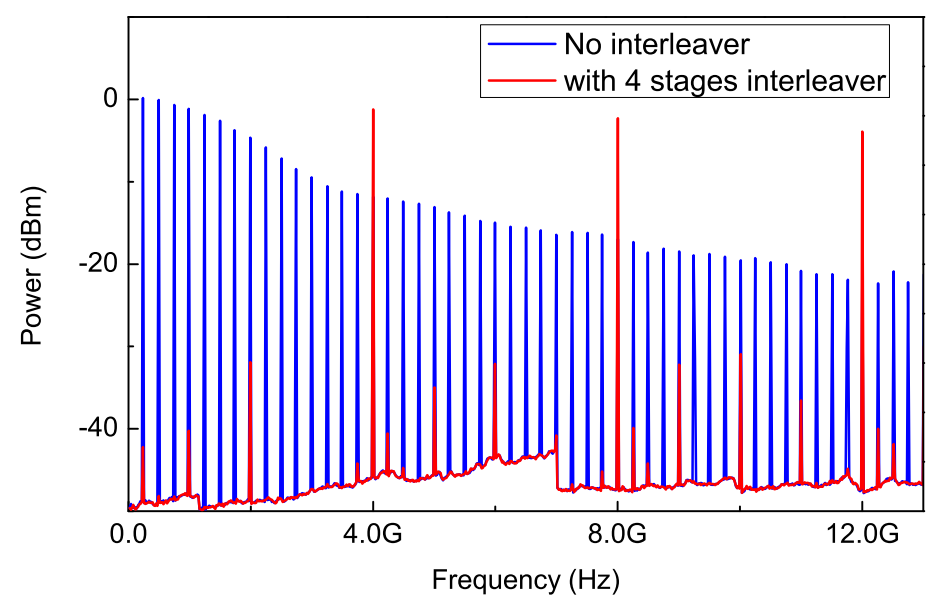

Figure 3. Photodiode output comparison with $10 \mathrm{dBm}$ optical power.

In order to improve the signal-to-noise ratio, a four stages fiber-based Mach-Zehnder interleaver is used at the output of optical frequency comb. ${ }^{19}$ Figures 3 displays the electrical PSD after the photodiode. A high linear photodiode with state-of-the-art flicker phase noise, below $-140 f^{-1} \mathrm{dBc} / \mathrm{Hz}$, where $f$ is the Fourier frequency offset, is used to convert optical pulses to electrical waveforms.

Compressing the optical pulse duration being photodetected as short as possible, even when it is shorter than the response time of photodiode, is essential to get phase noise result below $-173 \mathrm{dBc} / \mathrm{Hz}$ at high frequency offset. First, the photocurrent shot noise projection on phase is reduced by compressing the optical pulse duration. ${ }^{20}$ Second, we demonstrate the optical pulse duration fluctuation can convert into the phase fluctuation of microwave signal due to the nonlinearity of photodetection recently. ${ }^{21}$ Compressing the optical pulse duration will compress its fluctuation at the same time. Thus, decreasing the phase noise that contributed from the nonlinear conversion of optical pulse duration fluctuation. As tens of meters fiber (SMF28) are connected between the optical frequency comb and photodiode, we use the dispersion competent fiber (DCF38) just before the photodiode to compress the optical pulse width. Pulse duration below $0.8 \mathrm{ps}$ is abstained by carefully modifying the fiber length.

Amplitude noise to phase noise conversion (AM-PM) of photodetection of ultra-short optical pulse trains is another limitation to get ultra-low phase noise result. ${ }^{22,23}$ To overcome this limitation, we can optimize the RIN of the comb laser with EDFA saturation effect as mentioned above. We can also employ an active servo loop like reported in Ref. 23 to limit the RIN and stabilize the AM-PM. Furthermore, the AM-PM conversion coefficient depends on the working condition of photodiode like showing in Fig. 4. By setting suitable bias voltage and photocurrent, AM-PM rejection more than $30 \mathrm{~dB}$ and up to $50 \mathrm{~dB}$ are typically obtained and used.

\section{PHASE NOISE CHARACTERIZATION VIA DIGITAL CROSS CORRELATION}

Combing these forefront optoelectronics devices and cutting-edge techniques, microwave signals with a phase noise below $-173 \mathrm{dBc} / \mathrm{Hz}$ at $10 \mathrm{kHz}$ offset are made possible. However, characterization of such ultra-low phase 


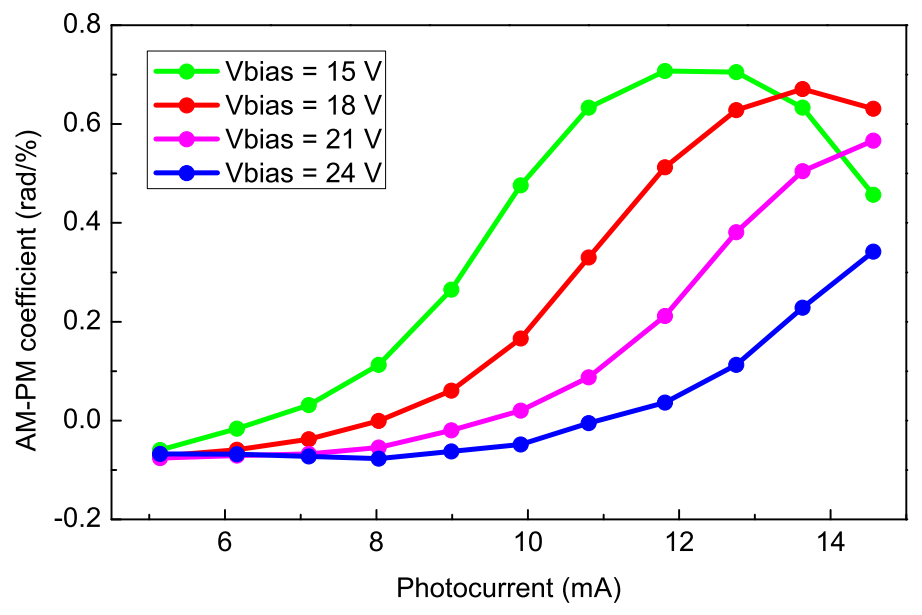

Figure 4. Amplitude noise to phase noise conversion of photodetection of ultra-short optical pulse trains.

noise is a challenge, even more so far high frequency carrier. Phase noise characterization is usually a process that involves comparing a signal from the device under test (DUT) with a reference source. When the signal under test has a lower phase noise than the available reference, two separate but identical systems can be built and compared. The data are then analysed assuming that the two identical systems contribute equally to the phase noise. However, to realize two equally good systems is not straightforward and a minute excess phase noise of the phase comparison system can strongly impact the final result obtained by such methods.

To overcome these limitations, we demonstrate a heterodyne digital cross-correlation scheme based on three similar but independent optoelectronic microwave-generation systems. ${ }^{21,24}$ By frequency mixing the DUT 12 $\mathrm{GHz}$ microwave signal with signals from the two auxiliary systems, two beat notes around $5 \mathrm{MHz}$ are obtained. These beat note signals, both of which carry phase information from the DUT, are sent to a field-programmable gate array (FPGA)-based heterodyne cross-correlator where they are sampled by fast analog-to-digital converters, digitally downconverted and processed to generate two independent phase comparison data sets. ${ }^{24}$ Crosscorrelation of these two phase-noise data sets converges to the absolute phase-noise PSD of the $12 \mathrm{GHz}$ signal that we want to characterize. The microwave signals generated by the two auxiliary systems act as phase references but do not need to be as good as the signal being characterized. Their uncorrelated noise determines the uncertainty of the estimates of the phase-noise PSD. ${ }^{25}$ The measurement noise floor is below $-180 \mathrm{dBc} / \mathrm{Hz}$ for Fourier frequencies beyond $1 \mathrm{kHz}$ offset.

Figure 5 displays the absolute phase noise PSD of generated $12 \mathrm{GHz}$. The phase noise of the $12 \mathrm{GHz}$ signal sticks almost entirely to the optical phase noise of the reference laser. It indicates a close-to-complete absolute transfer of spectral purity form optics to microwave.

\section{SUMMARY}

In conclusion, $12 \mathrm{GHz}$ microwave signals with unprecedented phase noise at both close and far Fourier frequencies are generated with an optical frequency comb. These phase noise results are successfully characterized by a digital cross correlation system. It paves the way to compact, ${ }^{26}$ robust and mobile microwave sources with ultra-low phase noise based on reliable technologies that have become readily accessible.

\section{ACKNOWLEDGMENTS}

We thank J. Pinto for help with the electronics and R. Le Targat for the reference-laser distribution. This work is funded by the Defense Advanced Research Projects Agency (DARPA) as a part of the Program in Ultrafast Laser Science and Engineering (P $\mu$ reComb project) under contract no. W31P4Q-14-C-0050, by the Formation, 


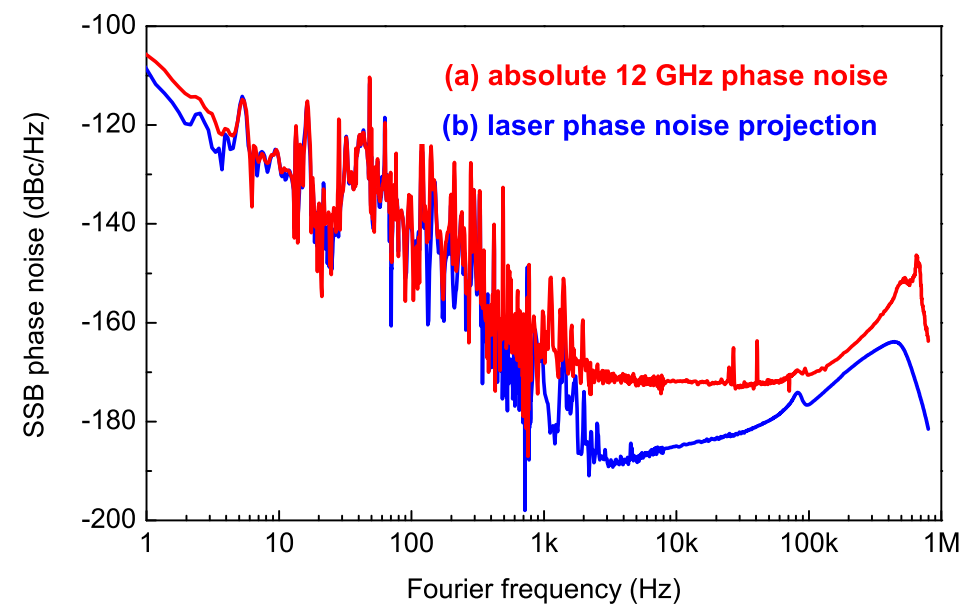

Figure 5. Absolute transfer of spectral purity from optics to microwave.

Innovation, Recherche, Services et Transfert en Temps-Fréquence (FIRST-TF) Labex, by the Eurostar Eureka program (Stable Microwave Generation and Dissemination over Optical Fiber project) and by the EU FP7 Initial Training Network FACT (Future Atomic Clock Technology).

\section{REFERENCES}

[1] Ghelfi, P., Laghezza, F., Scotti, F., Serafino, G., Capria, A., Pinna, S., Onori, D., Porzi, C., Scaffardi, M., Malacarne, A., et al., "A fully photonics-based coherent radar system," Nature 507(7492), 341-345 (2014).

[2] Koenig, S., Lopez-Diaz, D., Antes, J., Boes, F., Henneberger, R., Leuther, A., Tessmann, A., Schmogrow, R., Hillerkuss, D., Palmer, R., et al., "Wireless sub-thz communication system with high data rate," Nat. Photon. 7(12), 977-981 (2013).

[3] Millo, J., Abgrall, M., Lours, M., English, E., Jiang, H., Guéna, J., Clairon, A., Tobar, M., Bize, S., Le Coq, Y., et al., "Ultralow noise microwave generation with fiber-based optical frequency comb and application to atomic fountain clock," Appl. Phys. Lett. 94(14), 141105 (2009).

[4] Santarelli, G., Laurent, P., Lemonde, P., Clairon, A., Mann, A. G., Chang, S., Luiten, A. N., and Salomon, C., "Quantum projection noise in an atomic fountain: A high stability cesium frequency standard," Phys. Rev. Lett. 82(23), 4619 (1999).

[5] Maleki, L., "Sources: The optoelectronic oscillator," Nat. Photon. 5(12), 728-730 (2011).

[6] Li, J., Lee, H., and Vahala, K. J., "Microwave synthesizer using an on-chip brillouin oscillator," Nat. commun. 4, 2097 (2013).

[7] Schneider, G. J., Murakowski, J. A., Schuetz, C. A., Shi, S., and Prather, D. W., "Radiofrequency signalgeneration system with over seven octaves of continuous tuning," Nat. Photon. 7(2), 118-122 (2013).

[8] Li, J., Yi, X., Lee, H., Diddams, S. A., and Vahala, K. J., "Electro-optical frequency division and stable microwave synthesis," Science 345(6194), 309-313 (2014).

[9] Liang, W., Eliyahu, D., Ilchenko, V., Savchenkov, A., Matsko, A., Seidel, D., and Maleki, L., "High spectral purity kerr frequency comb radio frequency photonic oscillator," Nat. commun. 6 (2015).

[10] Zhang, W., Xu, Z., Lours, M., Boudot, R., Kersalé, Y., Santarelli, G., and Le Coq, Y., "Sub-100 attoseconds stability optics-to-microwave synchronization," Appl. Phys. Lett. 96(21), 211105 (2010).

[11] Fortier, T. M., Kirchner, M. S., Quinlan, F., Taylor, J., Bergquist, J., Rosenband, T., Lemke, N., Ludlow, A., Jiang, Y., Oates, C., et al., "Generation of ultrastable microwaves via optical frequency division," Nat. Photon. 5(7), 425-429 (2011). 
[12] Didier, A., Millo, J., Grop, S., Dubois, B., Bigler, E., Rubiola, E., Lacroûte, C., and Kersalé, Y., "Ultralow phase noise all-optical microwave generation setup based on commercial devices," Appl. Opt. 54(12), 3682-3686 (2015).

[13] Portuondo-Campa, E., Buchs, G., Kundermann, S., Balet, L., and Lecomte, S., "Ultra-low phase-noise microwave generation using a diode-pumped solid-state laser based frequency comb and a polarizationmaintaining pulse interleaver," Opt. Express 23(25), 32441-32451 (2015).

[14] Xie, X., Bouchand, R., Nicolodi, D., Giunta, M., Hänsel, W., Lezius, M., Joshi, A., Datta, S., Alexandre, C., Lours, M., et al., "Photonic microwave signals with zeptosecond-level absolute timing noise," Nat. Photon. 11(1), 44-47 (2017).

[15] Hänsch, T. W., "Nobel lecture: Passion for precision," Rev. Mod. Phys. 78, 1297-1309 (Nov 2006).

[16] Hall, J. L., "Nobel lecture: Defining and measuring optical frequencies," Rev. Mod. Phys. 78, 1279-1295 (Nov 2006).

[17] Xie, X., Bouchand, R., Nicolodi, D., Lours, M., Alexandre, C., and Coq, Y. L., "Phase noise characterization of sub-hertz linewidth lasers via digital cross correlation," Opt. Lett. 42, 1217-1220 (Apr 2017).

[18] Zhang, W., Lours, M., Fischer, M., Holzwarth, R., Santarelli, G., and Le Coq, Y., "Characterizing a fiberbased frequency comb with electro-optic modulator," IEEE transactions on ultrasonics, ferroelectrics, and frequency control 59(3), 432-438 (2012).

[19] Haboucha, A., Zhang, W., Li, T., Lours, M., Luiten, A. N., Coq, Y. L., and Santarelli, G., "Optical-fiber pulse rate multiplier for ultralow phase-noise signal generation," Opt. Lett. 36, 3654-3656 (Sep 2011).

[20] Quinlan, F., Fortier, T., Jiang, H., Hati, A., Nelson, C., Fu, Y., Campbell, J., and Diddams, S., "Exploiting shot noise correlations in the photodetection of ultrashort optical pulse trains," Nat. Photon. 7(4), 290 (2013).

[21] Xie, X., Bouchand, R., Nicolodi, D., Lours, M., Le Coq, Y., Tremblin, P.-A., Santarelli, G., Giunta, M., Hänsel, W., Lezius, M., et al., "Ultra-short optical pulses leading to ultra-stable photonic microwave generation," in [Frequency and Time Forum and IEEE International Frequency Control Symposium (EFTF/IFC), 2017 Joint Conference of the European], 781-783, IEEE (2017).

[22] Zhang, W., Li, T., Lours, M., Seidelin, S., Santarelli, G., and Le Coq, Y., "Amplitude to phase conversion of ingaas pin photo-diodes for femtosecond lasers microwave signal generation," Applied Physics B 106, 301-308 (Feb 2012).

[23] Bouchand, R., Nicolodi, D., Xie, X., Alexandre, C., and Coq, Y. L., "Accurate control of optoelectronic amplitude to phase noise conversion in photodetection of ultra-fast optical pulses," Opt. Express 25, 1226812281 (May 2017).

[24] Nicolodi, D., Bouchand, R., Lours, M., Xie, X., Le Coq, Y., Giunta, M., Lezius, M., Haensel, W., Holzwarth, R., Tremblin, P.-A., et al., "Ultra-low phase noise frequency-comb-based microwave generation and characterization," in [Frequency Control Symposium (IFCS), 2016 IEEE International], 1-3, IEEE (2016).

[25] Rubiola, E. and Giordano, V., "Correlation-based phase noise measurements," Rev. Sci. Instrum. 71(8), 3085-3091 (2000).

[26] Bouchand, R., Xie, X., Giunta, M., Hänsel, W., Lezius, M., Holzwarth, R., Alexandre, C., Tremblin, P.A., Santarelli, G., and Le Coq, Y., "Compact low-noise photonic microwave generation from commercial low-noise lasers," IEEE Photon. Techol. Lett. 29(16), 1403-1406 (2017). 